\title{
Measurements of the CKM angle $\gamma$ in tree-dominated decays at LHCb
}

\author{
Stefania Ricciardi ${ }^{1}$, a on behalf of the LHCb Collaboration \\ ${ }^{1}$ STFC Rutherford Appleton Laboratort, Chilton, Didcot, OX11 OQX
}

\begin{abstract}
We review the first measurements of the CKM angle $\gamma$ from LHCb. These measurements have been performed with $b$-hadron decays dominated by $b \rightarrow u$ and $b \rightarrow c$ tree-level amplitudes, from which $\gamma$ can be determined without theoretical uncertainties. Precision is achieved by averaging results from $B^{-} \rightarrow D h^{-}$ ( $h=K, \pi$ ) decays with $D \rightarrow h^{+} h^{-}, D \rightarrow K^{+} \pi^{-}$, and $D \rightarrow K^{+} \pi^{-} \pi^{+} \pi^{-}$, and $D \rightarrow K_{S}^{0} h^{+} h^{-}$. Prospects for these and future measurements of $\gamma$ using neutral $b$-hadron decays are briefly discussed.
\end{abstract}

\section{Introduction}

The measurement of the CKM angle $\gamma[\gamma=$ $\left.\arg \left(-\mathrm{V}_{\mathrm{ud}} \mathrm{V}_{\mathrm{ub}}^{*} / \mathrm{V}_{\mathrm{cd}} \mathrm{V}_{\mathrm{cb}}^{*}\right)\right]$ of the Unitarity Triangle (UT) is an important goal of the $\mathrm{LHCb}$ physics programme. The angle $\gamma$ plays a unique role among all $\mathrm{CP}$-violating parameters because it can be determined using pure tree-level decays of $B$ mesons without theoretical uncertainties. As tree-level measurements are expected to be insensitive to physics beyond the Standard Model (SM), the value of $\gamma$ determined in this way provides an important SM benchmark against which other measurements, more likely to be affected by phyics beyond the SM, can be compared. The power of this approach to new physics searches relies on precise measurements. Despite results from $B$-factories and Tevatron have improved considerably the knowledge of the UT angles and sides, $\gamma$ is still the least-well determined angle, with an experimental uncertainty from treelevel measurements of $9-12^{\circ}[1,2]$. This precision can be significantly improved with large datasets at $\mathrm{LHCb}$.

The angle $\gamma$ can be measured in a theoretically clean way by exploiting the interference between $b \rightarrow u$ and $b \rightarrow c$ tree-level transitions in decays of $b$-hadrons with a charm meson in the final state. Many $B \rightarrow D H$ decays are suitable, where $D$ indicates a $D^{0}$ or $\bar{D}^{0}$ and $H$ is the bachelor hadronic system, e.g., a kaon or a $K^{* 0}$ in the most sensitive measurements.

The first constraints on $\gamma$ at LHCb have been obtained from time-integrated measurements of charged $B$ mesons [3], which do not require flavour-tagging, therefore can exploit the full statistical power of the large $b \bar{b}$ production cross-section in $p p$ collisions at the LHC. In these measurements $\gamma$ is determined from the interference between $B^{+} \rightarrow D^{0} h^{+}$and $B^{+} \rightarrow \bar{D}^{0} h^{+}(h=K, \pi)^{1}$, where $D^{0}$ and $\bar{D}^{0}$ decay to a common final state. In addition to $\gamma$, the interference depends on two $B$ hadronic parameters,

\footnotetext{
a e-mail: stefania.ricciardi@stfc.ac.uk

${ }^{1}$ Charge-conjugation is implied throughout this paper unless otherwise stated.
}

which are known as $\delta_{B}$, the relative strong phase between the two $B$ decay amplitudes, and $r_{B}$, the relative ratio of the suppressed over the favoured amplitude. Different methods exist to determine these and other $D$ hadronic parameters from data without theoretical uncertainties. Three types of $D$ decays have been studied by $\mathrm{LHCb}$, corresponding to three well-established methods to determine $\gamma$ and all the unknown parameters: the GLW method [4, 5]), which uses decays to $C P$ eigenstates $\left(D \rightarrow K^{+} K^{-}\right.$and $D \rightarrow \pi^{+} \pi^{-}$); the ADS method [6]), which uses decays to quasi-specific flavour eigenstates $\left(D \rightarrow K^{+} \pi^{-}\right.$and $D \rightarrow K^{+} \pi^{-} \pi^{+} \pi^{-}$); and the GGSZ method [7], which uses self-conjugate three-body final states $\left(D \rightarrow K_{\mathrm{S}}^{0} \pi^{+} \pi^{-}\right.$and $D \rightarrow K_{\mathrm{S}}^{0} K^{+} K^{-}$).

Time-integrated measurements can also be performed with neutral $b$-hadrons when the concerned decay is selftagged. This is the case of $B^{0} \rightarrow D K^{* 0}$, where the charge of the kaon from the $K^{* 0} \rightarrow K^{+} \pi^{-}$decay identifies the flavour of the neutral $B$ meson. Preliminary results on these decays have recently been presented by $\mathrm{LHCb}$ [8].

In addition, $\mathrm{LHCb}$ has recently performed the first time-dependent analysis of $B_{s}^{0} \rightarrow D_{s}^{\mp} K^{ \pm}$decays [9], which is sensitive to $\gamma-2 \beta_{s}\left[\beta_{s}=\arg \left(-\mathrm{V}_{\mathrm{ts}} \mathrm{V}_{\mathrm{tb}}^{*} / \mathrm{V}_{\mathrm{cs}} \mathrm{V}_{\mathrm{cb}}^{*}\right)\right]$.

All the presented results use the 2011 dataset, corresponding to an integrated luminosity of $1 \mathrm{fb}^{-1}$ collected at $\sqrt{s}=7 \mathrm{TeV}$.

\section{Measurements with charged B decays}

\subsection{ADS/GLW results}

Charged $B$ decays to $D h^{+}$with $D \rightarrow h^{+} h^{-}$, where each $h$ can be a kaon or a pion, are characterised by an easy topology and can be reconstructed with relatively high efficiency at LHCb [10]. A combined GLW and ADS analysis is performed using the CP-even eigenstates $D \rightarrow h^{+} h^{-}$ and the quasi-flavour-specific eigenstate $D \rightarrow K^{+} \pi^{-}$. The observables with sensitivity to $\gamma$ are constructed by taking ratio of yields, so that many systematic uncertainties 


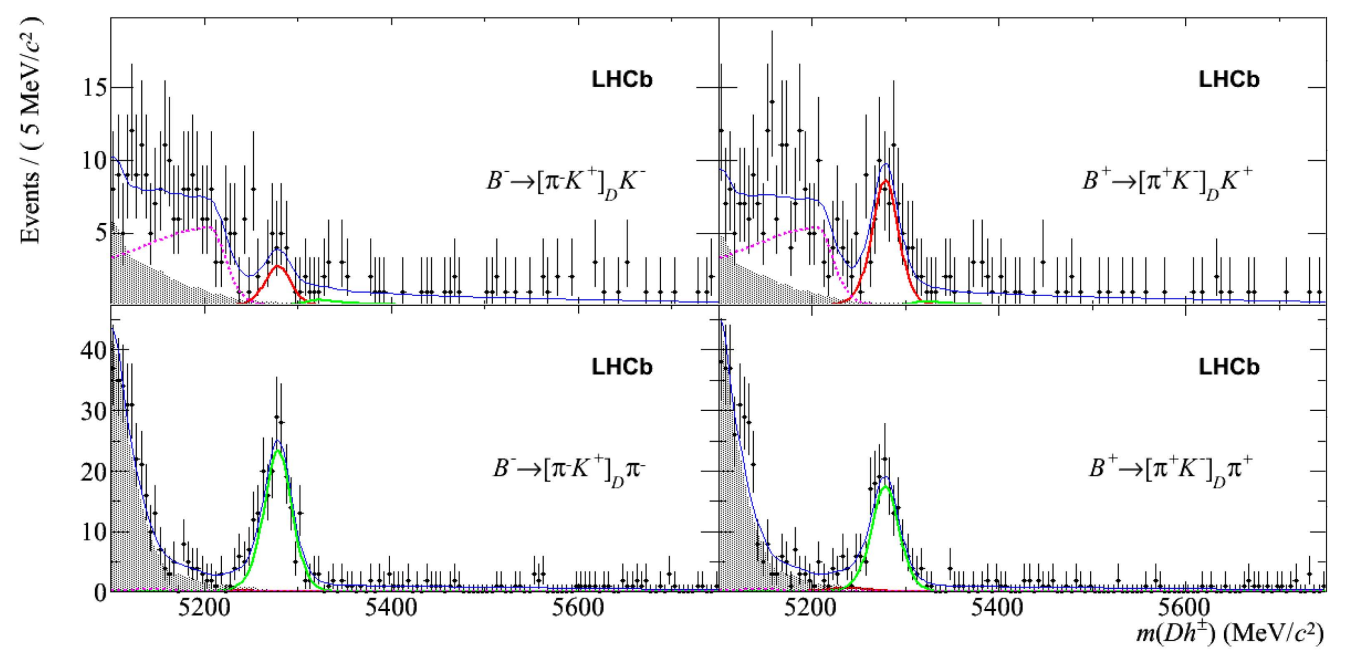

Figure 1. Mass distributions of the suppressed ADS decays: (left,top) $B^{-} \rightarrow D\left(K^{+} \pi^{-}\right) K^{-}$; (right,top) $B^{+} \rightarrow D\left(K^{+} \pi^{-}\right) K^{+}$; (left,bottom) $B^{-} \rightarrow D\left(K^{+} \pi^{-}\right) \pi^{-} ;$and (right,bottom) $B^{+} \rightarrow D\left(K^{+} \pi^{-}\right) \pi^{+}$.

cancel. $C P$ asymmetries and ratios of partial widths of suppressed over favoured modes are determined with a simultaneous fit to the $B$ mass of all 16 possible final states. The expected $C P$ asymmetries in the $B^{+} \rightarrow D \pi^{+}$channels are smaller than the corresponding ones in the $B^{+} \rightarrow D K^{+}$ channels, since the value of $r_{B}$, which controls the size of the $C P$ interference, is naively $\sim 20$ times smaller, but the large yields in the $B^{+} \rightarrow D \pi^{+}$channels help constrain the mass shape in the fit.

Among the results, we note the first evidence of nonzero $C P$ asymmetry, $A_{C P}$, between $B^{-} \rightarrow D K^{-}$and $B^{+} \rightarrow$ $D K^{+}$decays with $D \rightarrow K^{+} K^{-}, A_{C P}(K K)=0.148 \pm 0.037 \pm$ 0.010 , and $D \rightarrow \pi^{+} \pi^{-}, A_{C P}(\pi \pi)=0.135 \pm 0.066 \pm 0.010$, which combined give $4.5 \sigma$ significance for $C P$ violation in these modes. No significant asymmetry is found in the corresponding $B^{+} \rightarrow D \pi^{+}$modes.

Another important result of this analysis is the first observation of the rare $\mathrm{ADS}$ mode, $B^{+} \rightarrow D\left(K^{-} \pi^{+}\right) K^{+}$, with more than $10 \sigma$ significance. This mode is particularly sensitive to $\gamma$ since the two interfering amplitudes (i.e., the favoured $\bar{b} \rightarrow \bar{c}$ transition, followed by a doubly-Cabibbosuppressed $D$ decay, and the suppressed $\bar{b} \rightarrow \bar{u}$ transition, followed by the Cabibbo-favoured $D$ decay) have similar size, hence can give large asymmetries. The invariant mass distribution of both the suppressed $B^{+} \rightarrow D K^{+}$ and $B^{+} \rightarrow D \pi^{+}$modes, separated by $B$ charge, are shown in Fig. 1. There is evidence for large $C P$ asymmetry in the $B^{+} \rightarrow D K^{+}$mode, $A_{A D S}^{K}(K \pi)=-0.52 \pm 0.15 \pm 0.02$, and a hint of asymmetry in the $B^{+} \rightarrow D \pi^{+}, A_{A D S}^{\pi}(K \pi)=$ $0.143 \pm 0.062 \pm 0.011$. Further results on GLW and ADS observables are given in Ref. [10].

More recently, $\mathrm{LHCb}$ has published measurements of $C P$ observables using the $B^{+} \rightarrow D\left(K^{\mp} \pi^{ \pm} \pi^{\mp} \pi^{ \pm}\right) h^{+}$decay [11]. The ADS observables share the same $B$ decay parameters $\left(r_{B}, \delta_{B}\right)$ with $B^{+} \rightarrow D\left(K^{\mp} \pi^{ \pm}\right) h^{+}$, but different $D$ decay parameters, therefore, the inclusion of this decay adds complementary information, not just additional statistics, to the determination of $\gamma$ from a combined fit to all the modes. We note that the suppressed ADS modes in both the $B^{+} \rightarrow D K^{+}$and $B^{+} \rightarrow D \pi^{+}$channels have not previously been observed. In addition to the first observation of these modes at $\mathrm{LHCb}$, with a significance which exceeds $5 \sigma$ and $10 \sigma$, respectively, we mention a hint of $C P$ asymmetry, $A_{A D S}^{K}(K 3 \pi)=-0.42 \pm 0.22$ in the $B^{+} \rightarrow D K^{+}$ mode. The asymmetry in the $B^{+} \rightarrow D \pi^{+}$mode is measured to be $A_{A D S}^{\pi}(K 3 \pi)=0.13 \pm 0.10$.

\subsection{GGSZ results}

The GGSZ method exploits the different interference pattern, for $B^{+} \rightarrow D K^{+}$and $B^{-} \rightarrow D K^{-}$decays, in the $D \rightarrow$ $K_{\mathrm{s}}^{0} h^{+} h^{-}$Dalitz plot. This is a powerful method which dominates the sensitivity on $\gamma$ at $B$-factories, thanks to the rich resonance structure and the relatively large branching fraction of the $D \rightarrow K_{\mathrm{s}}^{0} \pi^{+} \pi^{-}$decay. The determination of $\gamma$ requires external information on the variation of the $D \rightarrow K_{\mathrm{s}}^{0} h^{+} h^{-}$amplitude phase over the Dalitz plot, $\delta_{D}$. A model-independent approach is taken in the first $\mathrm{LHCb}$ analysis with this method, which uses the CLEO [12] measurements of $\delta_{D}$ in bins of the Dalitz plot. The cartesian coordinates $x_{ \pm}=r_{B} \cos \left(\delta_{B} \pm \gamma\right)$ and $y_{ \pm}=r_{B} \sin \left(\delta_{B} \pm \gamma\right)$, are then extracted from a simultaneous fit to the $B^{ \pm}$mass distribution in each Dalitz-plot bin. The signal yields in each bin, $N\left(B^{ \pm}\right)_{i}$, are related to the cartesian coordinates by the following relations

$N\left(B^{+}\right)_{ \pm i} \propto K_{\mp i}+\left(x_{+}^{2}+y_{+}^{2}\right) K_{ \pm i}+2 \sqrt{K_{+i} K_{-i}}\left(x_{+} c_{ \pm i} \mp y_{+} s_{ \pm i}\right)$,

and

$N\left(B^{-}\right)_{ \pm i} \propto K_{ \pm i}+\left(x_{-}^{2}+y_{-}^{2}\right) K_{\mp i}+2 \sqrt{K_{+i} K_{-i}}\left(x_{-} c_{ \pm i} \pm y_{-} s_{ \pm i}\right)$

where $c_{i}$ and $s_{i}$ are the amplitude weighted average cosine and sine of the strong phase difference between the $D^{0}$ and $\bar{D}^{0}$ decay in bin $i$, and $K_{i}$ is the number of events in bin $i$ of a flavour-tagged $D^{0} \rightarrow K_{\mathrm{S}}^{0} \pi^{+} \pi^{-}$Dalitz plot. The in$\operatorname{dex} \pm i$ varies over the number of bins, $\pm i= \pm 1, \pm 8( \pm 2)$ 

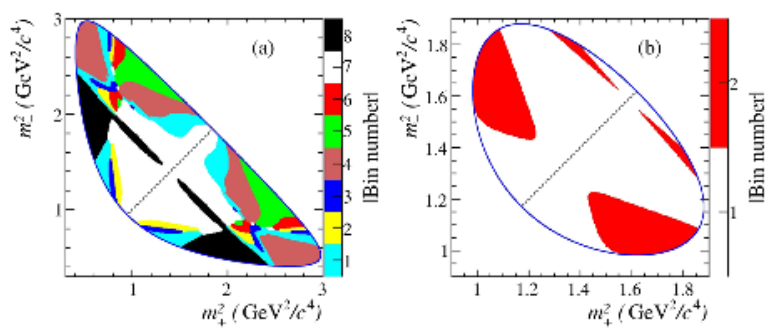

Figure 2. The Dalitz plot binning shemes for (a) $D \rightarrow K_{\mathrm{s}}^{0} \pi^{+} \pi^{-}$ and (b) $D \rightarrow K_{\mathrm{S}}^{0} K^{+} K^{-}$decays.

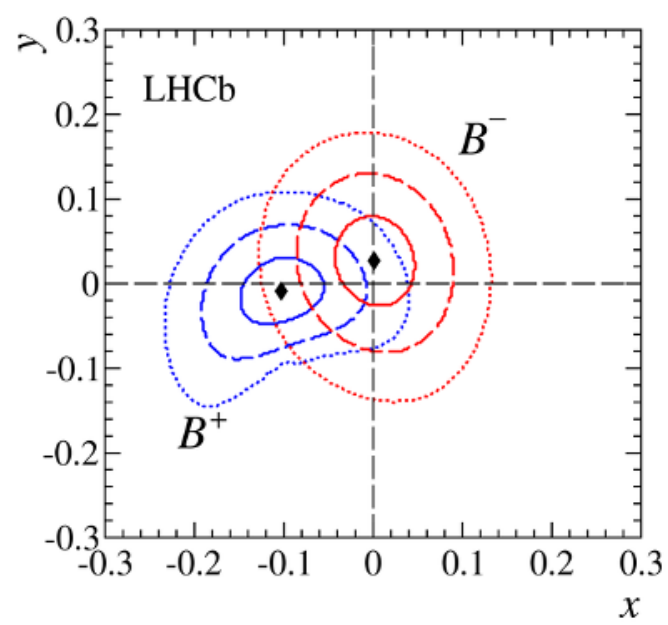

Figure 3. Cartesian coordinates results for (blue) $B^{+} \rightarrow$ $D\left(K_{\mathrm{S}}^{0} h^{+} h^{-}\right) K^{+}$and (red) $B^{-} \rightarrow D\left(K_{\mathrm{S}}^{0} h^{+} h^{-}\right) K^{-}$: (solid) one, (dashed) two, and (dotted) three standard deviation confidence levels (statistical only). The points represent the best fit central values.

for $D \rightarrow K_{\mathrm{s}}^{0} \pi^{+} \pi^{-}\left(D \rightarrow K_{\mathrm{S}}^{0} K^{+} K^{-}\right)$. The chosen binning schemes are shown in Fig. 2. The observed $B^{+} \rightarrow D K^{+}$ yields are 690 for $D \rightarrow K_{\mathrm{s}}^{0} \pi^{+} \pi^{-}$and $110 D \rightarrow K_{\mathrm{s}}^{0} K^{+} K^{-}$. In this analysis, the $B^{ \pm} \rightarrow D \pi^{ \pm}$modes are used not only to constrain the $B \rightarrow D K$ mass shape in the fit, but are also used to determine the variation in the reconstruction efficiency over the Dalitz plot. To do so the assumption of no $C P$ violation in these decays is made and a systematic uncertainty is assigned.

The best fit values for $x_{ \pm}$and $y_{ \pm}$are given in Ref. [13] and shown in Fig. 3 together with 1, 2, $3 \sigma$ contours for the statistical uncertainty which are obtained from the likelihood scan. A non-zero value of the angle between the two vectors joining the origin in the $x-y$ plane and the best fit values is a signature of $C P$ violation. The current data are compatible with both the $C P$ violation hypothesis and its absence. A frequentist approach is used to determine $\gamma, r_{B}$ and $\delta_{B}$ from the results on $x_{ \pm}$and $y_{ \pm}$. The solution for the physics parameters has a two-fold ambiguity, $(\gamma$, $\left.\delta_{B}\right)$ and $\left(\gamma+180^{\circ}, \delta_{B}+180^{\circ}\right)$. The solution that satisfies $0<\gamma<180^{\circ}$ is $\gamma=\left(44_{-38}^{+43}\right)^{\circ}, r_{B}=0.07 \pm 0.04$ and $\delta_{B}=\left(137_{-46}^{+35}\right)^{\circ}$. The value of $r_{B}$ is consistent with, but lower than, the world average of results from previous experiments [14]. This low value and its large correlation with the other physics parameters, also visible in Fig. 4, explain the large uncertainty on the values of $\gamma$ obtained from this analysis. More stringent contraints are obtained when these results are combined with the GLW and ADS measurements, which have complementary sensitivity to the same physics parameters.

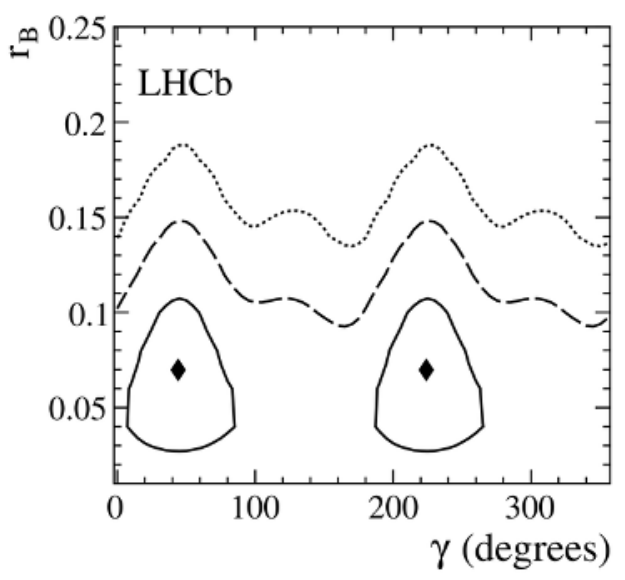

Figure 4. Two-dimensional projection of confidence regions onto the $\left(\gamma, r_{B}\right)$ plane showing the one (solid), two (dashed), three (dotted) standard deviation contours from the GGSZ method alone. The point marks are the central values.

\section{The LHCb $\gamma$ average}

Precision on $\gamma$ is achieved by combining all the results obtained with charged $B$ decays that are mentioned in Sect. 2 . A frequentist approach has been used by LHCb [3]. The combination uses additional inputs from CLEO [15] for the $D \rightarrow K^{+} \pi^{-}$and $D \rightarrow K^{+} \pi^{+} \pi^{-} \pi^{+}$decay parameters. The recent evidence for a difference in the $C P$ asymmetries in $D \rightarrow K^{+} K^{-}$and $D \rightarrow \pi^{+} \pi^{-}, \Delta a_{C P}^{d i r}=(-0.656 \pm 0.154) \times$ $10^{-2}$ [16], is taken into account in the combination, however it has only marginal effects on the final results.

The combination of all the $\mathrm{LHCb}$ results from $B^{+} \rightarrow$ $D K^{+}$decays gives at $68 \%$ C.L.

$$
\begin{gathered}
\gamma=71.1_{-15.7}^{+16.6}\left({ }^{\circ}\right), \\
r_{B}=0.092 \pm 0.008, \\
\delta_{B}=112.0_{-15.5}^{+12.6}\left({ }^{\circ}\right) .
\end{gathered}
$$

Both the central value of $\gamma$ and its uncertainty are in good agreement with the averages recently published by BaBar [17] and Belle [18]. While results from $B^{+} \rightarrow D K^{+}$ exhibit approximately Gaussian behaviour, as shown in Fig, 5, a double-peaked structure in the $\gamma$ 1-C.L. plot arises when the GLW/ADS results for $B^{+} \rightarrow D \pi^{+}$are included in the combination, as shown in Fig. 6. We set the confidence limits of

$$
\gamma \in[61.8,67.8]^{\circ} \text { or }[77.9,92.4]^{\circ} \text { at } 68 \% \text { C.L. },
$$




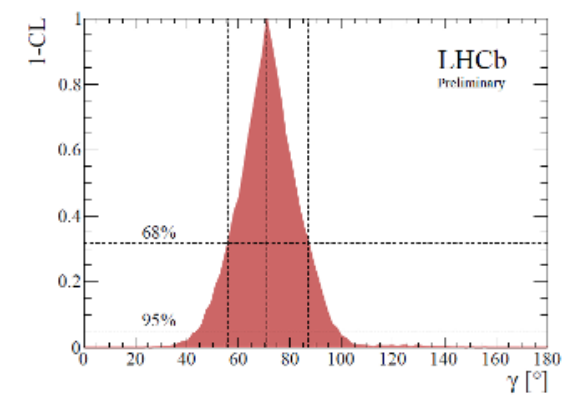

Figure 5. 1-C.L. curve for $\gamma$ for the combination of the measurements using $B^{+} \rightarrow D K^{+}$decays.

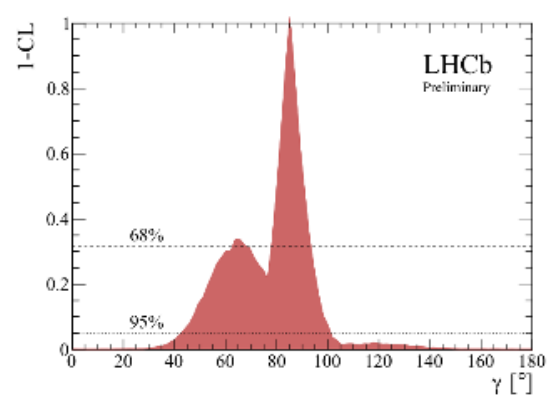

Figure 6. 1-C.L. curve for $\gamma$ for the combination of the measurements using both $B^{+} \rightarrow D K^{+}$and $B^{+} \rightarrow D \pi^{+}$decays.

$$
\gamma \in[43.8,101.5]^{\circ} \text { at } 95 \% \text { C.L., }
$$

where all values are modulo $180^{\circ}$. We note that the best fit value shifts to $85^{\circ}$ but the $95 \%$ confidence level is essentially unchanged. Despite the combined value is less than $1 \sigma$ from the $B^{+} \rightarrow D K^{+}$best fit value, the impact of $B^{+} \rightarrow D \pi^{+}$channel in the combination is probably larger than naively expected, and corresponds to rather high values of $r_{B}\left(D \pi^{+}\right)(\in[0.010-0.024]$ at $64 \%$ C.L. $)$, which are preferred by the data. $\mathrm{LHCb}$ is the only experiment which has included $B^{+} \rightarrow D \pi^{+}$results in the $\gamma$ average. Understanding and eventually fully exploiting $B^{+} \rightarrow D \pi^{+}$decays will be investigated with the analysis of the 2012 dataset.

\section{Measurements with $B^{0}$ decays}

The use of $B^{0} \rightarrow D K^{* 0}$ decays is particularly interesting because $r_{B}$ for this mode is naively a factor $\sim 3$ larger than the analogous ratio for $B^{+} \rightarrow D K^{+}$decays, hence the system can exhibit large $C P$-violating effects. The invariant mass distribution of the selected $D\left(K^{+} K^{-}\right) K^{* 0}$ candidates is shown in Fig. 7. In addition to the $B^{0}$ mass peak, also a peak at the $B_{s}^{0}$ mass is observed. The $\bar{B}_{s}^{0} \rightarrow D K^{* 0}$ decay is used as control channel, as no large $C P$ violation is expected in this mode. The $C P$ asymmetries, computed from the efficiency-corrected signal yields, are found to be

$$
\begin{gathered}
A_{C P}^{d}=-0.45 \pm 0.23 \pm 0.02, \\
A_{C P}^{s}=0.04 \pm 0.16 \pm 0.01,
\end{gathered}
$$
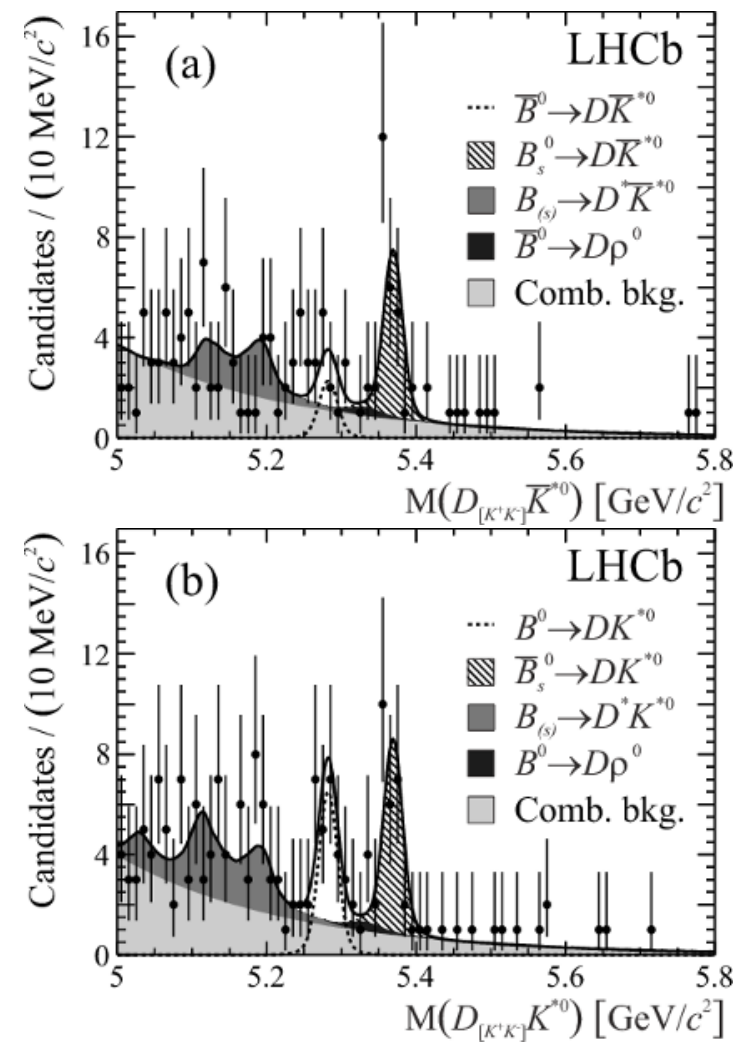

Figure 7. Invariant mass distributions of (a) $D_{\left[K^{+} K^{-}\right]} \bar{K}^{* 0}$ and (b) $D_{\left[K^{+} K^{-}\right]} K^{* 0}$ candidates. The fit functions are superimposed.

for $B^{0}$ and $\bar{B}_{s}^{0}$, respectively. Other results for the GLW observables can be found in Ref. [8]. These are the first measurements of $C P$ asymmetries in $B^{0}$ and $\bar{B}_{s}^{0}$ to $D K^{* 0}$ decays with the neutral $D$ meson decaying into a $C P$-even final state. With more data, improved measurements of these and other quantities in $B^{0} \rightarrow D K^{* 0}$ decays will set important constraints on $\gamma$.

\section{Time-dependent measurements in the $B_{s}^{0}$ system}

Interference effects are expected to be large also in the tree-level decays $B_{s}^{0} \rightarrow D_{s}^{\mp} K^{ \pm}$, since the $B_{s}^{0} \rightarrow D_{s}^{\mp} K^{ \pm}$and $\bar{B}_{s}^{0} \rightarrow D_{s}^{\mp} K^{ \pm}$interfering amplitudes are of the same order in the Wolfenstein parameter $\lambda, O\left(\lambda^{3}\right)$. A time-dependent analysis is performed as the two final states of interest are accessible by both $B_{s}^{0}$ and $\bar{B}_{s}^{0}$. The decay time-evolution is sensitive to $\gamma-2 \beta_{s}$, where $2 \beta_{s}$ is the $B_{s}^{0}$ mixing phase, which is small in the SM and is measured with $B_{s}^{0} \rightarrow J \psi \phi$ decays.

The first preliminary measurement of time-dependent $C P$ violation observables in $B_{s}^{0} \rightarrow D_{s}^{\mp} K^{ \pm}$decays has recently been reported by $\mathrm{LHCb}$ [9]. This measurement is unique to $\mathrm{LHCb}$, because it is the only existing experiment which has accumulated a sufficiently large sample of $B_{s}^{0}$ decays and is able to distinguish the rapid $B_{s}^{0}-\bar{B}_{s}^{0}$ oscillations (thanks to its excellent proper-time resolution and the boost of the $B_{s}^{0}$ mesons at LHC). Additional ex- 


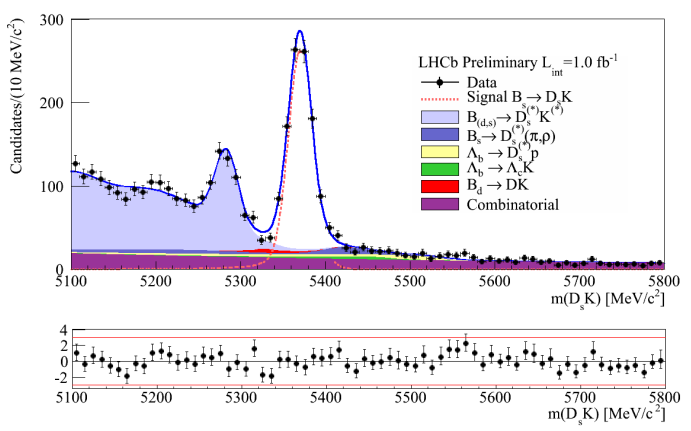

Figure 8. Results of the mass fit to $B_{s}^{0} \rightarrow D_{s}^{+} K^{-}$candidates.

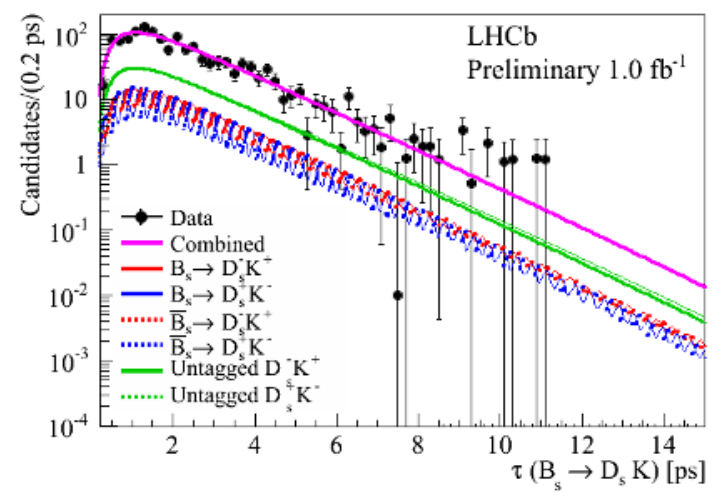

Figure 9. Results of the proper-time fit to $B_{s}^{0} \rightarrow D_{s}^{+} K^{-}$candidates. The top magenta curve represents the result of the combined fit to all the samples. The green solid (dashed) curve represents the result for the untagged $D_{s}^{-} K^{+}\left(D_{s}^{+} K^{-}\right)$sample. The four flavour-tagged samples are represented with red and blue curves corresponding to $D_{s}^{-} K^{+}$and $D_{s}^{+} K^{-}$, respectively; solid (dashed) lines are used for candidates tagged as $B_{s}^{0}\left(\bar{B}_{s}^{0}\right)$ at production.

perimental challenges for this measurement are related to the efficiency of the flavour-tagging algorithms and the determination of the proper-time acceptance. The LHCb results include both untagged and flavour-tagged candidates. Untagged events carry additional sensitivity to the weak phase since the decay width difference in the $B_{s}^{0}$ system, $\Delta \Gamma_{s}$, is sizeably different from zero. The mass distribution of the total sample is shown in Fig. 8, and the propertime distribution in Fig. 9, where the contribution of all the different components to the combined sample can be appreciated. The results on the $C P$ observables can be found in [9]. The correlations between the systematic uncertainties have an important impact on the determination of $\gamma-2 \beta_{s}$ and require further studies. No attempt has been made at this stage to derive confidence intervals for the weak phase.

\section{Conclusions and prospects}

Using $1 \mathrm{fb}^{-1}$ of the 2011 dataset, LHCb obtains $\gamma=$ $71.1_{-15.7}^{+16.6}\left(^{\circ}\right)$ by combining GLW, ADS and GGSZ $B^{+} \rightarrow$ $D K^{+}$observables. This result is in good agreement and has comparable precision to the combined results recently published by BaBar [17] and Belle [18]. Time-integrated measurements with $B^{0} \rightarrow D K^{* 0}$ and time-dependent measurement with $B_{s}^{0} \rightarrow D_{s}^{ \pm} K^{\mp}$ decays are also showing promising results. Statistical uncertainties are the dominant uncertainties in all these measurements. Precision on $\gamma$ is expected to improve significantly in the next years using these and other tree-level decays, which will become accessible with larger datasets. It has been estimated that sub-degree precision can be achieved with $50 \mathrm{fb}^{-1}$ at the proposed $\mathrm{LHCb}$ upgrade using a combination of several decay channels [19].

\section{Acknowledgements}

I would like to thank the organisers of HCP2012 for the excellent conference and warm hospitality in beautiful Kyoto.

\section{References}

[1] J. Charles et al. (CKMfitter Group), Eur.Phys.J. C41, 1 (2005), updated results and plots available at: http://ckmfitter.in2p3.fr

[2] D. Derkach (UTfit Collaboration), arXiv:1301.3300 (2013)

[3] R. Aaij et al. (LHCb collaboration), LHCb-CONF2012-032 (2012)

[4] M. Gronau, D. London, Phys.Lett. B253, 483 (1991)

[5] M. Gronau, D. Wyler, Phys.Lett. B265, 172 (1991)

[6] D. Atwood, I. Dunietz, A. Soni, Phys.Rev.Lett. 78, 3257 (1997)

[7] A. Giri, Y. Grossman, A. Soffer, J. Zupan, Phys.Rev. D68, 054018 (2003)

[8] R. Aaij et al. (LHCb collaboration) (2012), arXiv: 1212.5205

[9] R. Aaij et al. (LHCb collaboration), LHCb-CONF2012-029 (2012)

[10] R. Aaij et al. (LHCb Collaboration), Phys.Lett. B712, 203 (2012), erratum Phys. Lett. B713 (2012) 351

[11] R. Aaij et al. (LHCb collaboration), LHCb-CONF2012-030 (2012)

[12] J. Libby et al. (CLEO Collaboration), Phys.Rev. D82, 112006 (2010)

[13] R. Aaij et al., Phys. Lett. B718, 43 (2012)

[14] J. Beringer et al. (Particle Data Group), Phys.Rev. D86, 010001 (2012)

[15] N. Lowrey et al. (CLEO Collaboration), Phys.Rev. D80, 031105 (2009)

[16] Y. Amhis et al. (Heavy Flavor Averaging Group) (2012), arXiv: 1207.1158

[17] J. Lees et al. (BABAR Collaboration) (2013), arXiv: 1301.1029

[18] K. Trabelsi (Belle collaboration) (2013), arXiv: 1301.2033

[19] R. Aaij et al. (LHCb Collaboration) (2012), arXiv: 1208.3355 